\title{
Dynamics Simulation of Cam Mechanism Based on Pro/E
}

\author{
Liu Deliang \\ Transportation Equipments and Ocean Engineering \\ College \\ Dalian Maritime University \\ Dlain, China \\ e-mail: liudeliang@dlmu.edu.cn
}

\author{
Lu Huibiao \\ Transportation Equipments and Ocean Engineering \\ College \\ Dalian Maritime University \\ Dlain, China \\ e-mail:1hm_an@163.com
}

\author{
Cao Shuhua \\ Transportation Equipments and Ocean Engineering \\ College \\ Dalian Maritime University \\ Dlain, China \\ e-mail: daliankangbao@126.com \\ Sun Ang \\ Transportation Equipments and Ocean Engineering \\ College \\ Dalian Maritime University \\ Dlain, China \\ e-mail: sunangdl772@sina.com
}

\begin{abstract}
Cam mechanism is widely used as a common mechanism in automated or semi-automated machinery. To improve the cam mechanism design, efficiency and quality of machining process, a direct-acting roller follower disk cam mechanism was designed and simulated based on PRO/E. Computer-aided design and simulation methods of Cam mechanism were introduced, the movement of mechanism was very intuitive and the curve of each power parameter was available. The simulation allows designers to grasp and control the movement of mechanism and accuracy, increases design safety factor. The validity and reliability of the design were tested by comparing the results of the design and simulation analysis.
\end{abstract}

Keywords- cam mechanism; dynamics; simulation; CAD; reliability

\section{INTRODUCTION}

Cam mechanism is widely used as a common mechanism in automated or semi-automated machinery. Its advantages are compact, high-precision indexing, smooth transmission, good dynamic characteristics, small shock and vibration, big transmission torque, smooth movement and so on; its disadvantage is that the contact between the cam profile and follower is a point or line contact, easy to wear. So it often uses in the control mechanism that transmit force not great [1].

Depending on the shape of the cam mechanism can be divided into disc-shaped cam, movement cam and cylinder cam. According to the type of cam follower mechanism can be divided into the spire follower, roller follower and peaceful end follower.

Cam mechanism consists of three main components, cam, follower and rack. Cam mechanism is easy to design, that the movement principle of the driven element can be obtained only requires the design of appropriate cam profile. so the key of cam design is to get the cam profile contour surfaces.
In recent years, with the widespread application and development of three-dimensional CAD / CAE / CAM technology, the efficiency and quality of cam mechanism design and manufacture has greatly improved [2-5]. With Kinematics and dynamics simulation of cam mechanism, the mechanism movement can be observed very intuitive, and follower displacement, velocity, acceleration motion curve can be drawn, so that designers can grasp and control the movement of bodies and accuracy, increasing design safety factor [6-7].

In this paper, direct-acting roller follower disk cam mechanism is design and simulation with PRO / E software, computer-aided cam mechanism design and simulation methods are introduced, design solutions and reliability are tested by analysis and comparing the design and of simulation results.

\section{DETERMINATION OF THE CAM WORK PROFILE}

Design requirements: direct-acting roller follower disk cam mechanism, cam is rotated counterclockwise in equal angular velocity, offset $\mathrm{e}=10 \mathrm{~mm}$, cam base circle radius $r_{0}=60 \mathrm{~mm}$, roller radius $r_{t}=10 \mathrm{~mm}$, follower lift $\mathrm{h}=30 \mathrm{~mm}$, pushing away movement angle $\Phi=180^{\circ}$, far repose angle $\Phi_{\mathrm{s}}=30^{\circ}$, return movement angle $\Phi^{\prime}=120^{\circ}$, closed repose angle $\Phi_{\mathrm{s}}^{\prime}=60^{\circ}$, during pushing and return way, the follower both do simple harmonic motion.

Determining the movement of the follower according to job requirements, and then following the movement, the cam profile curve is designed, the cam working outline shown in Fig .1 could be got, the cam model shown in Fig .2 also could be established ${ }^{[8]}$. 


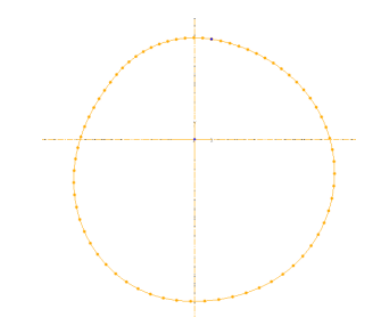

Figure 1. Cam work profile

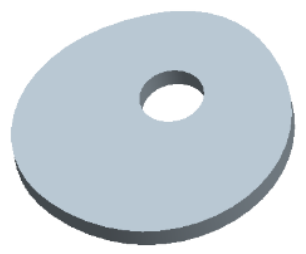

Figure 2. Cam model

\section{MOTION SIMULATION AND ANALYSIS OF CAM MECHANISM}

After the establishment of the cam, slide lever, roller, rack part model, using predefined connection conditions assembly in the PRO / E component modules, the assembly model shown in Fig .3 is established [9-10].

After the establishment of the assembly model, transferring into the agency work platform, adding the servo motor, the "Type" select "Connect axis", "Standard" choose "Speed", "initial position" is set to "0" , "model" select constant and input 6. Selecting the outer surface of the cam and the outer surface of the roller cam mechanism to establish the connection, the movement model shown in Fig .4 is established.

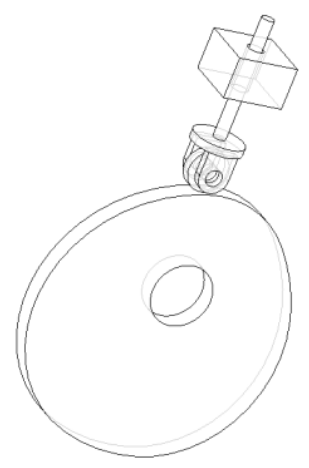

Figure 3. Cam mechanism model

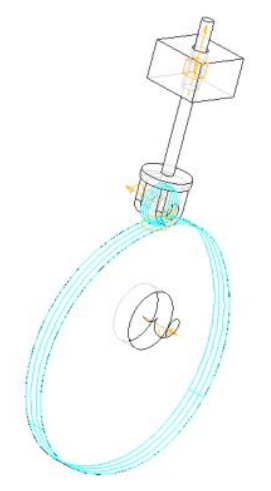

Figure 4. Cam motion model

Upon completion of the connection and drive, kinematic analysis is organized; the end time is set to 60, exactly one rotation of the cam, the motor choose the default pre-set motor.

In kinematics analysis, measuring the displacement, velocity and acceleration were selected in order and the displacement, velocity and acceleration curves were shown in Fig .5-7.
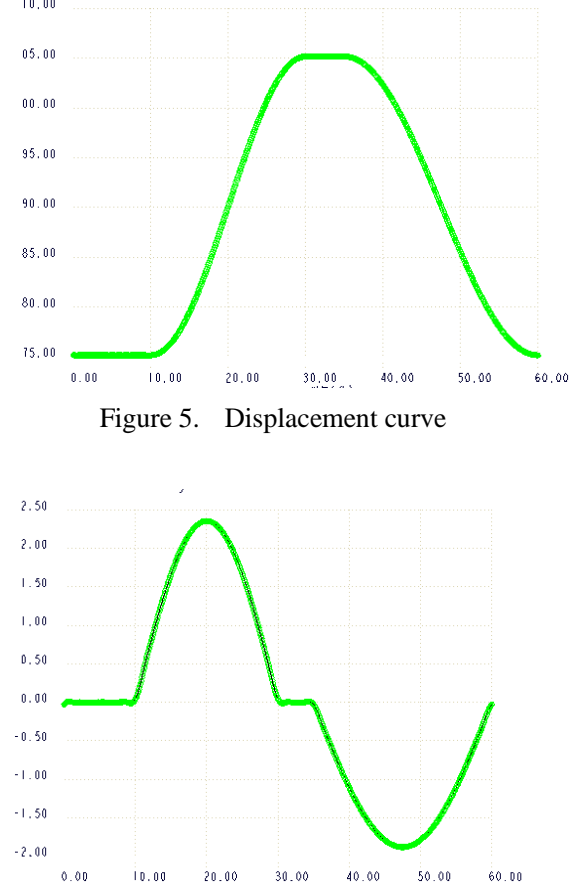

Figure 6. Velocity curve

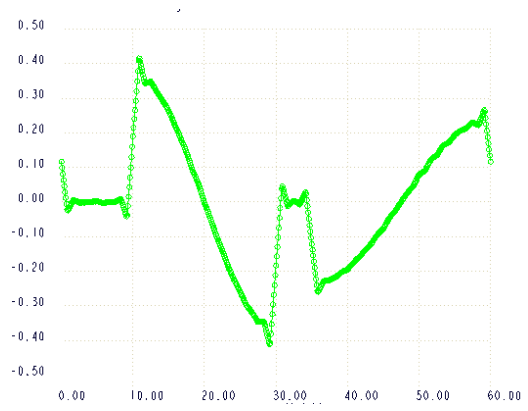

Figure 7. Acceleration curve 


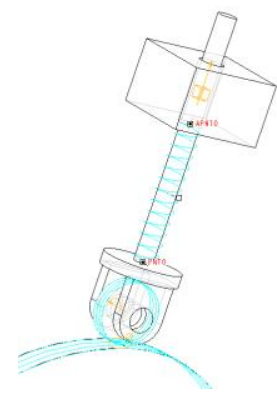

Figure 8. Spring model

Displacement, velocity and acceleration curves show that during $0-10$ seconds $\left(360^{\circ}-300^{\circ}\right)$ in the vertical direction follower is static, during 10-30 seconds $\left(300^{\circ}\right.$ $180^{\circ}$ ) follower does simple harmonic motion, the follower stroke $\mathrm{h}=30 \mathrm{~mm}$ or swing angle $\varphi=120^{\circ}$, during 30-35 seconds $\left(180^{\circ}-150^{\circ}\right)$ in the vertical direction follower is static, during 10-30 seconds $\left(150^{\circ}-0^{\circ}\right)$ follower does simple harmonic motion, the follower stroke $\mathrm{h}=30 \mathrm{~mm}$ or swing angle $\varphi=150^{\circ}$, regardless of displacement, velocity or acceleration curves are consistent with the theoretical design law.

\section{DYNAMICS SIMULATION ANALYSIS OF CAM MECHANISM}

\section{A. Adding quality attributes}

With "gravity tool" in "dynamic" toolbar, the mold for gravity is set as $9806.65 \mathrm{~mm} / \mathrm{sec} 2$, gravity direction is negative $\mathrm{Y}$ axis. With "quality attributes" tool in the "dynamic" toolbar, "density" options are defined, selecting the coordinate system to the local coordinate system LCS, in the basic properties options group giving the density as 7.82e-9tonne / mm3 (45 steel), in the inertia tab defining at the center of gravity, then the cam, slide lever, roller and rack quality attribute definitions are completed.

\section{B. Establishing a spring connection}

With the "spring" tool in the "dynamic" toolbar, each a point on slide bar and rack is selected as the start and end point of the spring, as shown in Fig .8. In the property options the spring elastic coefficient is defined as $0.5 \mathrm{~N} /$ $\mathrm{mm}$, spring original length is defined as $45 \mathrm{~mm}$; spring diameter is defined as $9 \mathrm{~mm}$.

\section{Defining dynamic analysis}

Upon completion of the connection and drive, clicking "institutional analysis" button, "analysis of the definition of" dialog box is pop-up, accepting the default name, in the "Type" drop-down list box choose "dynamic" option, in the "Preferences" tab, setting end time is 60s, exactly one rotation of the cam, the motor choose the default pre-set one, in the "external load" tab, checking the "enable gravity 'checkbox. Click the operation "run" to see bodies.

\section{Measurement Results}

In the dynamic analysis, the axis shaft stress, the axis largest shaft, motor torque, the maximum torque of the motor, the maximum pressure angle and the force of the spring are analyzed respectively in order. Assuming no friction between the cam and the follower roller, and no sliding, the cam roller contact force was carried out only, each of the respective measurement results are shown in following Fig.

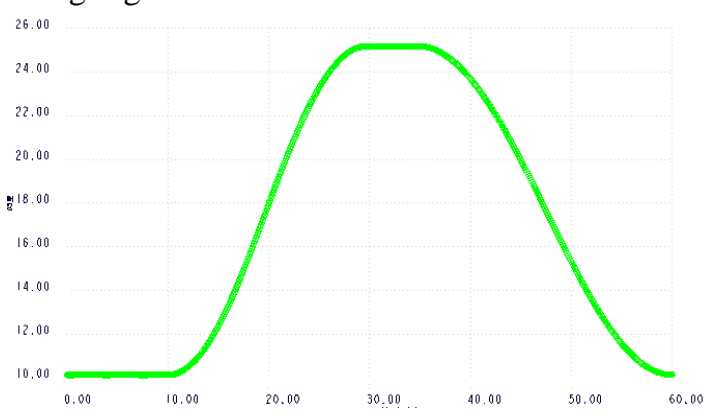

Figure 9. Acceleration curve

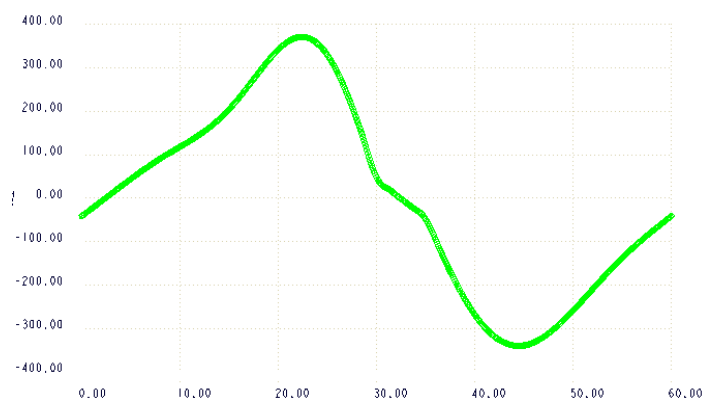

Figure 10. Spring model
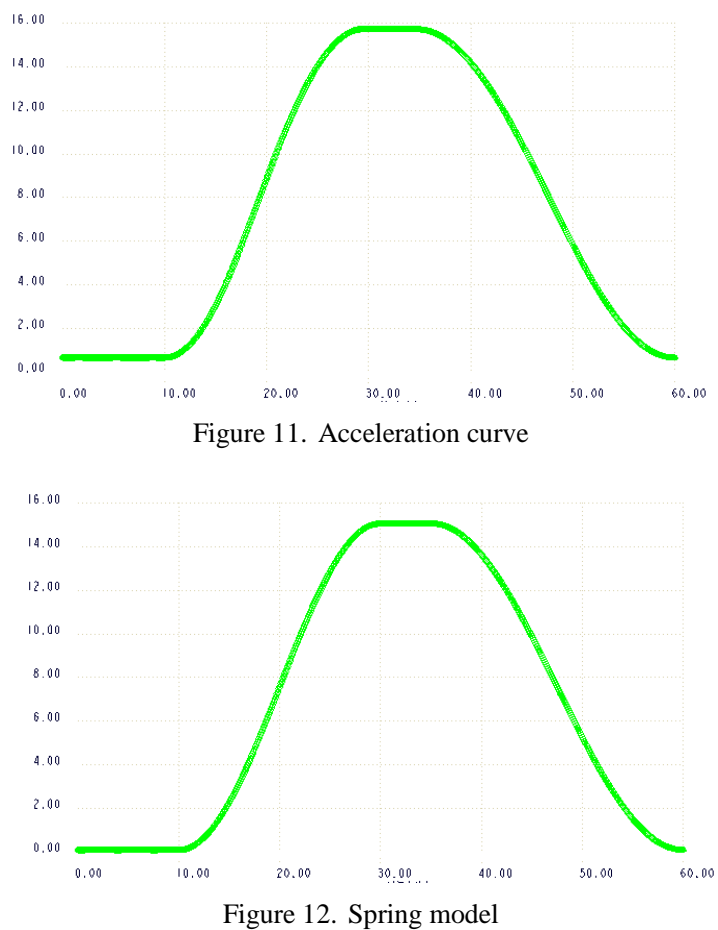

\section{CONCLUSION}

Motion simulation and dynamic analysis is a procedure of establishing a virtual mechanism designed on a computer to achieve the purpose of simulating real bodies moving in a virtual environment. It has a significant role to improve Design efficiency, reduce costs and shorten the design cycle. For the cam mechanism widely used in automated or semi-automated machinery, in order to improve the design, processing efficiency and quality, it 
was designed and simulated with PRO / E software, computer-aided cam mechanism design and simulation methods are introduced, the movement of bodies was very visually observe, and the power curve of each parameter was got, so that designers can grasp and control the movement of bodies and precision, increasing the safety factor. Design solutions correctness and reliability are tested by analysis and comparing the design and of simulation results.

\section{REFERENCES}

[1] Yang Kezheng, Cheng Guanyun, Li Hongsheng. Fundamentals of Machine Design. Higher Education Press. 2005, 10.

[2] Yin Guofu, Yang Suixian. Computer-aided design and manufacturing technology. Huazhong University of Science and Technology Press. 2008.09.

[3] Jin Xiaoyi, Zhang Minliang. Software Development for Motion Analysis of Disc Cam Mechanism Based on Precision measurement. 2011 Third International Conference on Measuring Technology and Mechatronics Automation. 302-305.

[4] Xiaoming Han Yucheng Bo Huiyuan Wang Jian Xu. Dynamics Analysis of Cam Drive Mechanism with Clearance. 2011 IEEE 2nd International Conference on Computing, Control and Industrial Engineering, v 1, p 345-347.

[5] XianHai Yang, Wang Wang. Fuzzy Optimization Design and Motion Simulation for Conjugate Cam Mechanism. Proceedings of the IEEE International Conference on Automation and Logistics.2066-2068.August 18 - 21, 2007, Jinan, China.

[6] Yin Ming-Fu,Zhao Zhen-Hong. Study on One-side Machining Principle and Tool Path Control Method of the Globoidal Cam. China MechanicalEngineering, 2005, 02, 127-130.

[7] Ge Zhengtao, Yang Fuliang. Pro/ENGINEER Wildfire 3.0 motion and dynamic simulation analysis. 2007.08

[8] Tang Zhaoping, Sun Jianping. The parametric design and motion analysis of disc cam mechanism with straight motion and deviation falted-faced follower. Manufacturing Automation , 2011 , VOL.33 No. 4 : 105-108。

[9] Liao Haiping, Liu Qiyue, Zeng Cuihua, Ren Hongbing. Flat translating follower disk cam mechanism EHL design. JOURNAL OF MACHINE DESIGN, 2009, VOL.26 No.5 : 71-72。 .

[10] Herman J. Van de Straete and Joris De Schutter. Hybrid Cam Mechanism. IEEE/ASME TRANSACTIONS ON MECHATRONICS, VOL. 1, NO. 4, 284-289, DECEMBER 1996. 